\title{
Research Article \\ On Coincidence and Fixed-Point Theorems in Symmetric Spaces
}

\author{
Seong-Hoon Cho, ${ }^{1}$ Gwang-Yeon Lee, ${ }^{1}$ and Jong-Sook Bae ${ }^{2}$ \\ ${ }^{1}$ Department of Mathematics, Hanseo University, Chungnam 356-706, South Korea \\ ${ }^{2}$ Department of Mathematics, Moyngji University, Youngin 449-800, South Korea
}

Correspondence should be addressed to Seong-Hoon Cho, shcho@hanseo.ac.kr

Received 28 August 2007; Revised 4 February 2008; Accepted 5 March 2008

Recommended by Lech Gorniewicz

We give an axiom (C.C) in symmetric spaces and investigate the relationships between (C.C) and axioms (W3), (W4), and (H.E). We give some results on coinsidence and fixed-point theorems in symmetric spaces, and also, we give some examples for the results of Imdad et al. (2006).

Copyright (C) 2008 Seong-Hoon Cho et al. This is an open access article distributed under the Creative Commons Attribution License, which permits unrestricted use, distribution, and reproduction in any medium, provided the original work is properly cited.

\section{Introduction}

In [1], the author introduced the notion of compatible mappings in metric spaces and proved some fixed-point theorems. This concept of compatible mappings was frequently used to show the existence of common fixed points. However, the study of the existence of common fixed points for noncompatible mappings is, also, very interesting. In [2], the author initially proved some common fixed-point theorems for noncompatible mappings. In [3], the authors gave a notion (E-A) which generalizes the concept of noncompatible mappings in metric spaces, and they proved some common fixed-point theorems for noncompatible mappings under strict contractive conditions. In [4], the authors proved some common fixed-point theorems for strict contractive noncompatible mappings in metric spaces. Recently, in [5] the authors extended the results of $[3,4]$ to symmetric(semimetric) spaces under tight conditions. In [6], the author gave a common fixed-point theorem for noncompatible self-mappings in a symmetric spaces under a contractive condition of integral type.

In this paper, we give some common fixed-point theorems in symmetric(semimetric) spaces and give counterexamples for the results of Imdad et al. [5].

In order to obtain common fixed-point theorems in symmetric spaces, some axioms are needed. In [5], the authors assumed axiom (W3), and in [6] the author assumed axioms (W3), (W4), and (H.E); see Section 2 for definitions. 
We give another axiom for symmetric spaces and study their relationships in Section 2. We give common fixed-point theorems of four mappings in symmetric spaces and give some examples which justifies the necessity of axioms in Section 3.

\section{Axioms on symmetric spaces}

A symmetric on a set $X$ is a function $d: X \times X \rightarrow[0, \infty)$ satisfying the following conditions:

(i) $d(x, y)=0$, if and only if $x=y$ for $x, y \in X$,

(ii) $d(x, y)=d(y, x)$, for all $x, y \in X$.

Let $d$ be a symmetric on a set $X$. For $x \in X$ and $\epsilon>0$, let $B(x, \epsilon)=\{y \in X: d(x, y)<\epsilon\}$. A topology $\tau(d)$ on $X$ defined as follows: $U \in \tau(d)$ if and only if for each $x \in U$, there exists an $\epsilon>0$ such that $B(x, \epsilon) \subset U$. A subset $S$ of $X$ is a neighbourhood of $x \in X$ if there exists $U \in \tau(d)$ such that $x \in U \subset S$. A symmetric $d$ is a semimetric if for each $x \in X$ and each $\epsilon>0$, $B(x, \epsilon)$ is a neighbourhood of $x$ in the topology $\tau(d)$.

A symmetric (resp., semimetric) space $(X, d)$ is a topological space whose topology $\tau(d)$ on $X$ is induced by symmetric(resp., semi-metric) $d$.

The difference of a symmetric and a metric comes from the triangle inequality. Actually a symmetric space need not be Hausdorff. In order to obtain fixed-point theorems on a symmetric space, we need some additional axioms. The following axioms can be found in [7].

(W3) for a sequence $\left\{x_{n}\right\}$ in $X, x, y \in X, \lim _{n \rightarrow \infty} d\left(x_{n}, x\right)=0$ and $\lim _{n \rightarrow \infty} d\left(x_{n}, y\right)=0$ imply $x=y$.

(W4) for sequences $\left\{x_{n}\right\},\left\{y_{n}\right\}$ in $X$ and $x \in X, \lim _{n \rightarrow \infty} d\left(x_{n}, x\right)=0$ and $\lim _{n \rightarrow \infty} d\left(y_{n}, x_{n}\right)=$ 0 imply $\lim _{n \rightarrow \infty} d\left(y_{n}, x\right)=0$.

Also the following axiom can be found in [6].

(H.E) for sequences $\left\{x_{n}\right\},\left\{y_{n}\right\}$ in $X$ and $x \in X, \lim _{n \rightarrow \infty} d\left(x_{n}, x\right)=0$ and $\lim _{n \rightarrow \infty} d\left(y_{n}, x\right)=0$ imply $\lim _{n \rightarrow \infty} d\left(x_{n}, y_{n}\right)=0$.

Now, we add a new axiom which is related to the continuity of the symmetric $d$.

(C.C) for sequences $\left\{x_{n}\right\}$ in $X$ and $x, y \in X, \lim _{n \rightarrow \infty} d\left(x_{n}, x\right)=0$ implies $\lim _{n \rightarrow \infty} d\left(x_{n}, y\right)=d(x, y)$.

Note that if $d$ is a metric, then (W3), (W4), (H.E), and (C.C) are automatically satisfied. And if $\tau(d)$ is Hausdorff, then (W3) is satisfied.

Proposition 2.1. For axioms in symmetric space $(X, d)$, one has

(1) $(\mathrm{W} 4) \Rightarrow(\mathrm{W} 3)$,

(2) $($ C.C $) \Rightarrow(W 3)$.

Proof. Let $\left\{x_{n}\right\}$ be a sequence in $X$ and $x, y \in X$ with $\lim _{n \rightarrow \infty} d\left(x_{n}, x\right)=0$ and $\lim _{n \rightarrow \infty} d\left(x_{n}, y\right)=0$.

(1) By putting $y_{n}=y$ for each $n \in N$, we have $\lim _{n \rightarrow \infty} d\left(x_{n}, y_{n}\right)=\lim _{n \rightarrow \infty} d\left(x_{n}, y\right)=0$. By (W4), we have $0=\lim _{n \rightarrow \infty} d\left(y_{n}, x\right)=d(y, x)$.

(2) By (C.C), $\lim _{n \rightarrow \infty} d\left(x_{n}, x\right)=0$ implies $d(x, y)=\lim _{n \rightarrow \infty} d\left(x_{n}, y\right)=0$.

The following examples show that other relationships in Proposition 2.1 do not hold. 
Example 2.2. (W4) $\nRightarrow($ H.E) and (W4) $\nRightarrow($ C.C) and so (W3) $\nRightarrow($ H.E) and (W3) $\nRightarrow($ C.C) by Proposition $2.1(1)$.

Let $X=[0, \infty)$ and let

$$
d(x, y)= \begin{cases}|x-y| & (x \neq 0, y \neq 0), \\ \frac{1}{x} & (x \neq 0) .\end{cases}
$$

Then, $(X, d)$ is a symmetric space which satisfies (W4) but does not satisfy (H.E) for $x_{n}=n, y_{n}=n+1$. Also $(X, d)$ does not satisfy (C.C).

Example 2.3. (H.E) $\nRightarrow($ W3), and so (H.E) $\nRightarrow($ W4) and (H.E) $\nRightarrow($ C.C).

Let $X=[0,1] \cup\{2\}$ and let

$$
d(x, y)= \begin{cases}|x-y| & (0 \leq x \leq 1,0 \leq y \leq 1) \\ |x| & (0<x \leq 1, y=2)\end{cases}
$$

and $d(0,2)=1$.

Then, $(X, d)$ is a symmetric space which satisfies (H.E). Let $x_{n}=1 / n$. Then, $\lim _{n \rightarrow \infty} d\left(x_{n}, 0\right)=\lim _{n \rightarrow \infty} d\left(x_{n}, 2\right)=0$. But $d(0,2) \neq 0$ and hence the symmetric space $(X, d)$ does not satisfy (W3).

Example 2.4. (C.C) $\nRightarrow(\mathrm{W} 4)$ and so (W3) $\nRightarrow(\mathrm{W} 4)$ by Proposition 2.1(2).

Let $X=\{1 / n: n=1,2, \ldots\} \cup\{0\}$, and let $d(0,1 / n)=(1 / n)(n$ is odd $), d(0,1 / n)=1(n$ is even) and

$$
d\left(\frac{1}{m}, \frac{1}{n}\right)= \begin{cases}\left|\frac{1}{m}-\frac{1}{n}\right| & (m+n \text { is even }) \\ \left|\frac{1}{m}-\frac{1}{n}\right| & (m+n \text { is odd and }|m-n|=1) \\ 1 & (m+n \text { is odd and }|m-n|>2)\end{cases}
$$

Then, the symmetric space $(X, d)$ satisfies (C.C) but does not satisfy (W4) for $x_{n}=$ $1 /(2 n+1)$ and $y_{n}=1 / 2 n$.

Example 2.5. (C.C) $\nRightarrow($ H.E).

Let $X=\{1 / n: n=1,2, \ldots\} \cup\{0\}$, and let

$$
d\left(\frac{1}{m}, \frac{1}{n}\right)= \begin{cases}\left|\frac{1}{m}-\frac{1}{n}\right| & (|m-n| \geq 2), \\ 1 & (|m-n|=1)\end{cases}
$$

and $d(1 / n, 0)=1 / n$. Then, $(X, d)$ is a symmetric space which satisfies (C.C). Let $x_{n}=$ $1 / n, y_{n}=1 /(n+1)$. Then, $\lim _{n \rightarrow \infty} d\left(x_{n}, 0\right)=\lim _{n \rightarrow \infty} d\left(y_{n}, 0\right)=0$. But $\lim _{n \rightarrow \infty} d\left(x_{n}, y_{n}\right) \neq 0$. Hence, the symmetric space $(X, d)$ does not satisfy (H.E). 


\section{Common fixed points of four mappings}

Let $(X, d)$ be a symmetric (or semimetric) space and let $f, g$ be self-mappings of $X$. Then, we say that the pair $(f, g)$ satisfies property (E-A) [3] if there exists a sequence $\left\{x_{n}\right\}$ in $X$ and a point $t \in X$ such that $\lim _{n \rightarrow \infty} d\left(f x_{n}, t\right)=\lim _{n \rightarrow \infty} d\left(g x_{n}, t\right)=0$.

A subset $S$ of a symmetric space $(X, d)$ is said to be $d$-closed if for a sequence $\left\{x_{n}\right\}$ in $S$ and a point $x \in X, \lim _{n \rightarrow \infty} d\left(x_{n}, x\right)=0$ implies $x \in S$. For a symmetric space $(X, d)$, $d$-closedness implies $\tau(d)$-closedness, and if $d$ is a semimetric, the converse is also true.

At first, we prove coincidence point theorems of four mappings satisfying the property (E-A) under some contractive conditions.

Theorem 3.1. Let $(X, d)$ be a symmetric(semimetric) space that satisfies (W3) and (H.E), and let $A, B, S$, and $T$ be self-mappings of $X$ such that

(1) $A X \subset T X$ and $B X \subset S X$,

(2) the pair $(B, T)$ satisfies property $(E-A)$ (resp., $(A, S)$ satisfies property $(E-A))$,

(3) for any $x, y \in X, d(A x, B y) \leq m(x, y)$, where

$$
m(x, y)=\max \{d(S x, T y), \min \{d(A x, S x), d(B y, T y)\}, \min \{d(A x, T y), d(B y, S x)\}\},
$$

(4) SX is a d-closed ( $\tau(d)$-closed) subset of X (resp., TX is a d-closed $(\tau(d)$-closed) subset of $X)$.

Then, there exist $u, w \in X$ such that $A u=S u=B w=T w$.

Proof. From (2), there exist a sequence $\left\{x_{n}\right\}$ in $X$, and a point $t \in X$ such that $\lim _{n \rightarrow \infty} d\left(T x_{n}, t\right)=\lim _{n \rightarrow \infty} d\left(B x_{n}, t\right)=0$.

From (1), there exists a sequence $\left\{y_{n}\right\}$ in $X$ such that $B x_{n}=S y_{n}$ and hence $\lim _{n \rightarrow \infty} d\left(S y_{n}, t\right)=0$. By (H.E), $\lim _{n \rightarrow \infty} d\left(B x_{n}, T x_{n}\right)=\lim _{n \rightarrow \infty} d\left(S y_{n}, T x_{n}\right)=0$.

From (4), there exists a point $u \in X$ such that $S u=t$.

From (3), we have

$$
\begin{aligned}
& d\left(A u, B x_{n}\right) \\
& \quad \leq \max \left\{d\left(S u, T x_{n}\right), \min \left\{d(A u, S u), d\left(B x_{n}, T x_{n}\right)\right\}, \min \left\{d\left(A u, T x_{n}\right), d\left(B x_{n}, S u\right)\right\}\right\} .
\end{aligned}
$$

By taking $n \rightarrow \infty$, we have $\lim _{n \rightarrow \infty} d\left(A u, B x_{n}\right)=0$. By (W3), we get $A u=S u$.

Since $A X \subset T X$, there exists a point $w \in X$ such that $A u=T w$.

We show that $T w=B w$. From (3), we have

$$
\begin{aligned}
& d(A u, B w) \\
& \quad \leq \max \{d(S u, T w), \min \{d(A u, S u), d(B w, T w)\}, \min \{d(A u, T w), d(B w, S u)\}\} \\
& \quad=\max \{d(T w, T w), \min \{d(A u, A u), d(B w, T w)\}, \min \{d(A u, A u), d(B w, S u)\}\}=0 .
\end{aligned}
$$

Hence, $A u=B w$ and hence $A u=S u=B w=T w$.

For the existence of a common fixed point of four self-mappings of a symmetric space, we need an additional condition, so-called weak compatibility.

Recall that for self-mappings $f$ and $g$ of a set, the pair $(f, g)$ is said to be weakly compatible [8] if $f g x=g f x$, whenever $f x=g x$. Obviously, if $f$ and $g$ are commuting, the pair $(f, g)$ is weakly compatible. 
Theorem 3.2. Let $(X, d)$ be a symmetric(semimetric) space that satisfies (W3) and (H.E), and let $A, B, S$, and $T$ be self-mappings of $X$ such that

(1) $A X \subset T X$ and $B X \subset S X$,

(2) the pair $(B, T)$ satisfies property $(E-A)($ resp., $(A, S)$ satisfies property $(E-A))$,

(3) the pairs $(A, S)$ and $(B, T)$ are weakly compatible,

(4) for any $x, y \in X(x \neq y), d(A x, B y)<m(x, y)$,

(5) SX is a d-closed $(\tau(d)$-closed) subset of X (resp., TX is a d-closed ( $\tau(d)$-closed) subset of $X)$.

Then, $A, B, S$, and $T$ have a unique common fixed point in $X$.

Proof. From Theorem 3.1, there exist $u, w \in X$ such that $A u=S u=T w=B w$. From (3), $A S u=S A u, A A u=A S u=S A u=S S u$ and $B T w=T B w=T T w=B B w$.

If $A u \neq w$, then from (4) we have

$$
\begin{aligned}
d & (A u, A A u) \\
\quad & =d(A A u, B w) \\
& <\max \{d(S A u, T w), \min \{d(A A u, S A u), d(B w, T w)\}, \min \{d(A A u, T w), d(B w, S A u)\}\} \\
\quad & =\max \{d(A A u, A u), 0, d(A A u, A u)\} \\
\quad & =d(A A u, A u)
\end{aligned}
$$

which is a contradiction.

Similarly, if $u \neq B w$, we have a contradiction. Thus, $A u=w=S u=T w=B w=u$, and $w$ is a common fixed point of $A, B, S$, and $T$.

For the uniqueness, let $z$ be another common fixed point of $A, B, S$, and $T$. If $w \neq z$, then from (4) we get

$$
\begin{aligned}
d(z, w) & =d(A z, B w) \\
& <\max \{d(S z, T w), \min \{d(A z, S z), d(B w, T w)\}, \min \{d(A z, T w), d(B w, S z)\} \\
& =\max \{d(z, w), \min \{d(z, z), d(w, w)\}, \min \{d(z, w), d(w, z)\} \\
& =d(z, w)
\end{aligned}
$$

which is a contradiction. Hence, $w=z$.

Remark 3.3. In the case of $A=B=g$ and $S=T=f$ in Theorem 3.1 (resp., Theorem 3.2), we can show that $f$ and $g$ have a coincidence point (resp., $f$ and $g$ have a unique common fixed point) without making the assumption $g X \subset f X$.

Recently, R. P. Pant and V. Pant [4] obtained the existence of a common fixed point of the pair of $(f, g)$ in a metric space $(X, d)$ satisfying the condition

(P.P) for any $x, y \in X$,

$$
d(g x, g y)<\max \left\{d(f x, f y), \frac{k}{2}\{d(f x, g x)+d(f y, g y)\}, \frac{1}{2}\{d(f y, g x)+d(f x, g y)\}\right\},
$$

where $1 \leq k<2$. 
Also in [5], the authors tried to extend the result of [4] to symmetric spaces which satisfy axiom (W3).

Now, we will extend R. P. Pant and V. Pant's result to symmetric spaces which satisfy additional conditions (H.E) and (C.C).

Theorem 3.4. Let $(X, d)$ be a symmetric(semimetric) space that satisfies (H.E) and (C.C) and let $A, B, S$, and $T$ be self-mappings of $X$ such that

(1) $A X \subset T X$ and $B X \subset S X$,

(2) the pair $(B, T)$ satisfies property $(E-A)$ (resp., $(A, S)$ satisfies property $(E-A))$,

(3) for any $x, y \in X, d(A x, B y) \leq m_{1}(x, y)$, where $m_{1}(x, y)=\max \{d(S x, T y),(k / 2)$ $\{d(A x, S x)+d(B y, T y)\},(k / 2)\{d(A x, T y)+d(B y, S x)\}\}, 0<k<2$,

(4) SX is a d-closed $(\tau(d)$-closed) subset of X (resp., TX is a d-closed ( $\tau(d)$-closed) subset of $X)$.

Then, there exist $u, w \in X$ such that $A u=S u=B w=T w$.

Proof. As in the proof of Theorem 3.1, there exist sequences $\left\{x_{n}\right\},\left\{y_{n}\right\}$ in $X$ and a point $t \in X$ such that $\lim _{n \rightarrow \infty} d\left(T x_{n}, t\right)=\lim _{n \rightarrow \infty} d\left(B x_{n}, t\right)=0$ and $B x_{n}=S y_{n}$. Hence, $\lim _{n \rightarrow \infty} d\left(S y_{n}, t\right)=0$.

From (4), there exists a point $u \in X$ such that $S u=t$.

We show $A u=S u$. From (3), we have

$$
\begin{aligned}
& d\left(A u, B x_{n}\right) \\
& \quad \leq \max \left\{d\left(S u, T x_{n}\right), \frac{k}{2}\left\{d(A u, S u)+d\left(B x_{n}, T x_{n}\right)\right\}, \frac{k}{2}\left\{d\left(A u, T x_{n}\right)+d\left(B x_{n}, S u\right)\right\}\right\}
\end{aligned}
$$

In the above inequality, we take $n \rightarrow \infty$, by (C.C) and (H.E), we have

$$
\begin{aligned}
d(A u, S u) & \leq \max \left\{0, \frac{k}{2} d(A u, S u), \frac{k}{2}\{d(A u, S u)\}\right. \\
& =\frac{k}{2} d(A u, S u) .
\end{aligned}
$$

Since $0<k / 2<1$, we get $d(A u, S u)=0$ and hence $A u=S u$.

Since $A X \subset T X$, there exists a point $w \in X$ such that $A u=T w$.

We show that $T w=B w$. From (3), we have

$$
\begin{aligned}
d(T w, B w) & =d(A u, B w) \\
& \leq \max \left\{d(S u, T w), \frac{k}{2}\{d(A u, S u)+d(B w, T w)\}, \frac{k}{2}\{d(A u, T w)+d(B w, S u)\}\right\} \\
& =\max \left\{d(T w, T w), \frac{k}{2}\{d(A u, A u)+d(B w, T w)\}, \frac{k}{2}\{d(A u, A u)+d(B w, S u)\}\right\} \\
& =\max \left\{\frac{k}{2} d(B w, T w), \frac{k}{2} d(B w, S u)\right\} \\
& =\frac{k}{2} d(B w, T w) .
\end{aligned}
$$

Since $0<k / 2<1$, we get $d(T w, B w)=0$ and hence $T w=B w$. Therefore, we have $A u=S u=B w=T w$. 
Theorem 3.5. $(X, d)$ be a symmetric(semimetric) space that satisfies (H.E) and (C.C) and let $A, B, S$, and $T$ be self-mappings of $X$ such that

(1) $A X \subset T X$ and $B X \subset S X$,

(2) the pair $(B, T)$ satisfies property $(E-A)$ (resp., $(A, S)$ satisfies property $(E-A))$,

(3) the pairs $(A, S)$ and $(B, T)$ are weakly compatible,

(4) for any $x, y \in X(x \neq y), d(A x, B y)<m_{2}(x, y)$, where $m_{2}(x, y)=\max \{d(S x, T y)$, $(k / 2)\{d(A x, S x)+d(B y, T y)\},(1 / 2)\{d(A x, T y)+d(B y, S x)\}\}, 0<k<2$.

(5) $S X$ is a d-closed $(\tau(d)$-closed) subset of $X$ (resp., TX is a d-closed( $\tau(d)$-closed) subset of $X)$.

Then $A, B, S$, and $T$ have a unique common fixed point in $X$.

Proof. From Theorem 3.4, there exist points $u, w \in X$ such that $A u=S u=T w=B w, A A u=$ $A S u=S A u=S S u$, and $B T w=T B w=T T w=B B w$.

We show that $A u=w$. If $A u \neq w$, then from (4) we have

$d(A u, A A u)$

$=d(A A u, B w)$

$<\max \left\{d(S A u, T w), \frac{k}{2}\{d(A A u, S A u)+d(B w, T w)\}, \frac{1}{2}\{d(A A u, T w)+d(B w, S A u)\}\right\}$

$=\max \{d(A A u, A u), 0, d(A A u, A u)\}=d(A A u, A u)$.

which is a contradiction.

Similarly, if $u \neq B w$, we have a contradiction. Thus $A u=w=S u=T w=B w=u$.

For the uniqueness, let $w$ be another common fixed point of $A, B, S$, and $T$. If $w \neq z$, then from (4) we get

$$
\begin{aligned}
d(z, w) & =d(A z, B w) \\
& <\max \left\{d(S z, T w), \frac{k}{2}\{d(A z, S z)+d(B w, T w)\}, \frac{1}{2}\{d(A z, T w)+d(B w, S z)\}\right\} \\
& =\max \left\{d(z, w), \frac{k}{2}\{d(z, z)+d(w, w)\}, \frac{1}{2}\{d(z, w)+d(w, z)\}\right\} \\
& =\max \{d(z, w), 0, d(w, z)\}=d(z, w) .
\end{aligned}
$$

which is a contradiction. Hence $w=z$.

Example 3.6. Let $X=[0,1]$ and $d(x, y)=(x-y)^{2}$. Define self-mappings $A, B, S$, and $T$ by $A x=B x=(1 / 2) x$ and $S x=T x=x$ for all $x \in X$. Then, we have the following:

(0) $(X, d)$ is a symmetric space satisfying the properties (H.E) and (C.C),

(1) $A X \subset T X$ and $B X \subset S X$,

(2) the pair $(B, T)$ satisfies property (E-A) for the sequence $x_{n}=1 / n, n=1,2,3, \ldots$,

(3) the pairs $(A, S)$ and $(B, T)$ are weakly compatible,

(4) for any $x, y \in X(x \neq y), d(A x, B y)<d(S x, T y) \leq m_{i}(x, y), i=1,2$,

(5) $S X$ is a $d$-closed $(\tau(d)$-closed) subset of $X$,

(6) $A 0=B 0=S 0=T 0=0$. 
Remark 3.7. In the case of $A=B=g$ and $S=T=f$ in Theorem 3.4 (resp., Theorem 3.5), we can show that $f$ and $g$ have a coincidence point (resp., $f$ and $g$ have a unique common fixed point) without the condition (1), that is, $g X \subset f X$.

Theorem 3.4.

The following example shows that the axioms (H.E) and (C.C) cannot be dropped in

Example 3.8. Let $(X, d)$ be the symmetric space as in Example 2.2. Then, the symmetric $d$ does not satisfy both (H.E) and (C.C).

Let $S=T=f$ and $A=B=g$ be self-mappings of $X$ defined as follows:

$$
f x=x(x \geq 0), \quad g x= \begin{cases}\frac{1}{3} x & (x>0), \\ \frac{1}{3} & (x=0) .\end{cases}
$$

Then, the condition (3) (resp., (4)) of Theorem 3.4 (resp., Theorem 3.5) is satisfied for $k=1$.

To show this, let $n_{1}(x, y)=\max \{d(f x, f y),(1 / 2)\{d(f x, g x)+d(f y, g y)\},(1 / 2)\{d(f y$, $g x)+d(f x, g y)\}\}$. We consider two cases.

Case 1. $x=0, y>0$,

$$
\begin{aligned}
n_{1}(x, y) & =\max \left\{d(0, y), \frac{1}{2}\left\{d\left(0, \frac{1}{3}\right)+d\left(y, \frac{1}{3} y\right)\right\}, \frac{1}{2}\left\{d\left(0, \frac{1}{3} y\right)+d\left(y, \frac{1}{3}\right)\right\}\right\} \\
& =\max \left\{\frac{1}{y}, \frac{1}{2}\left\{3+\frac{2}{3} y\right\}, \frac{1}{2}\left\{\frac{3}{y}+\left|y-\frac{1}{3}\right|\right\}\right\} \\
& \geq \frac{1}{2}\left\{3+\frac{2}{3} y\right\}=\frac{y}{3}+\frac{3}{2}>\frac{1}{3}|y-1|=d\left(\frac{1}{3}, \frac{1}{3} y\right)=d(g x, g y) .
\end{aligned}
$$

Case 2. $x>0, y>0(x \neq y)$,

$$
n_{1}(x, y) \geq d(f x, f y)=|x-y|>\frac{1}{3}|x-y|=d(g x, g y) .
$$

Thus, the condition (3) (resp., (4)) of Theorem 3.4 (resp., Theorem 3.5) is satisfied. Note that $f X$ is a $d$-closed $(\tau(d)$-closed) subset of $X$. Also, the pair $(f, g)$ satisfies property (E-A) for $x_{n}=n$, but the pair $(f, g)$ has no coincidence points, and also the pair $(f, g)$ has no common fixed points.

Remark 3.9. Example 3.6 satisfies all conditions of [5, Theorems 2.1 and 2.2] and satisfies also all conditions of [5, Theorem 2.3].

Let $\phi: \mathbb{R}^{+} \rightarrow \mathbb{R}^{+}$be a function such that

$$
\begin{aligned}
& (\phi 1) \phi \text { is nondecreasing on } \mathbb{R}^{+}, \\
& (\phi 2) 0<\phi(t)<t \text { for all } t \in(0, \infty) .
\end{aligned}
$$

Note that from $(\phi 1)$ and $(\phi 2)$, we have $\phi(0)=0$.

On the studying of fixed points, various conditions of $\phi$ have been studied by many different authors $[3,5,6]$. 
Remark 3.10. The functions $m_{i}(x, y)$ in Theorems 3.4 and 3.5 can be generalized to the compositions $\phi\left(m_{i}(x, y)\right)$ for $i=1,2$.

Example 3.11. Let $(X, d)$ be the symmetric space and $A, B, S$, and $T$ be the functions as in Example 3.8. Recall that $(X, d)$ satisfies (W3) but does not satisfy both (H.E) and (C.C). Let $\phi(t)=(2 / 3) t, t \in \mathbb{R}^{+}$and $k=3 / 2$. Then, for any $x, y \in X, d(A x, B y) \leq \phi\left(m_{i}(x, y)\right)$ for $i=1,2$. Note that the pairs $(A, S)$ and $(B, T)$ satisfy property (E-A), and $A X \subset T X, B X \subset S X$, and $S X$ are $d$-closed $(\tau(d)$-closed).

Therefore, $A, B, S$, and $T$ satisfy all conditions of [5, Theorem 2.4] and satisfy also all conditions of [5, Theorem 2.5]. But the pairs $(A, S)$ and $(B, T)$ have no points of coincidence, and also the pairs $(A, S)$ and $(B, T)$ have no common fixed points.

\section{Acknowledgments}

The authors are very grateful to the referees for their helpful suggestions. The first author was supported by Hanseo University, 2007.

\section{References}

[1] G. Jungck, "Compatible mappings and common fixed points," International Journal of Mathematics and Mathematical Sciences, vol. 9, no. 4, pp. 771-779, 1986.

[2] R. P. Pant, "Common fixed points of noncommuting mappings," Journal of Mathematical Analysis and Applications, vol. 188, no. 2, pp. 436-440, 1994.

[3] M. Aamri and D. El Moutawakil, "Some new common fixed point theorems under strict contractive conditions," Journal of Mathematical Analysis and Applications, vol. 270, no. 1, pp. 181-188, 2002.

[4] R. P. Pant and V. Pant, "Common fixed points under strict contractive conditions," Journal of Mathematical Analysis and Applications, vol. 248, no. 1, pp. 327-332, 2000.

[5] M. Imdad, J. Ali, and L. Khan, "Coincidence and fixed points in symmetric spaces under strict contractions," Journal of Mathematical Analysis and Applications, vol. 320, no. 1, pp. 352-360, 2006.

[6] A. Aliouche, "A common fixed point theorem for weakly compatible mappings in symmetric spaces satisfying a contractive condition of integral type," Journal of Mathematical Analysis and Applications, vol. 322, no. 2, pp. 796-802, 2006.

[7] W. A. Wilson, "On semi-metric spaces," American Journal of Mathematics, vol. 53, no. 2, pp. 361-373, 1931.

[8] G. Jungck, "Common fixed points for noncontinuous nonself maps on nonmetric spaces," Far East Journal of Mathematical Sciences, vol. 4, no. 2, pp. 199-215, 1996. 